\title{
RNA interference gene therapy in dominant retinitis pigmentosa and cone-rod dystrophy mouse models caused by GCAP1 mutations
}

\author{
Li Jiang $^{1}{ }^{*}$, Jeanne M. Frederick ${ }^{1}$ and Wolfgang Baehr ${ }^{1,2,3}$ * \\ ' Department of Ophthalmology and Visual Sciences, John A. Moran Eye Center, University of Utah Health Science Center, Salt Lake City, UT, USA \\ ${ }^{2}$ Department of Biology, University of Utah, Salt Lake City, UT, USA \\ ${ }^{3}$ Department of Neurobiology and Anatomy, University of Utah Health Science Center, Salt Lake City, UT, USA
}

\section{Edited by:}

Rameshwar K. Sharma, Salus

University, USA

\section{Reviewed by:}

Karl-Wilhelm Koch, Carl von Ossietzky University Oldenburg, Germany

James Ames, University of California, Davis, USA

Alexander Dizhoor, Salus University, USA

\section{${ }^{*}$ Correspondence:}

Li Jiang and Wolfgang Baehr.

Department of Ophthalmology and Visual Sciences, John A. Moran Eye Center, University of Utah Health Science Center, 65 Mario Capecchi Drive, Salt Lake City, UT 84132, USA e-mail: li.jiang@hsc.utah.edu; wbaehr@hsc.utah.edu
RNA interference (RNAi) knockdown is an efficacious therapeutic strategy for silencing genes causative for dominant retinal dystrophies. To test this, we used self-complementary (sc) AAV2/8 vector to develop an RNAi-based therapy in two dominant retinal degeneration mouse models. The allele-specific model expresses transgenic bovine GCAP1(Y99C) establishing a rapid RP-like phenotype, whereas the nonallele-specific model expresses mouse GCAP1 $(\mathrm{L} 151 \mathrm{~F})$ producing a slowly progressing cone-rod dystrophy (CORD). The late onset GCAP1(L151F)-CORD mimics the dystrophy observed in human GCAP1-CORD patients. Subretinal injection of scAAV2/8 carrying shRNA expression cassettes specific for bovine or mouse guanylate cyclase-activating protein 1 (GCAP1) showed strong expression at 1 week post-injection. In both allele-specific [GCAP1(Y99C)-RP] and nonallelespecific [GCAP1 (L151F)-CORD] models of dominant retinal dystrophy, RNAi-mediated gene silencing enhanced photoreceptor survival, delayed onset of degeneration and improved visual function. Such results provide a "proof of concept" toward effective RNAi-based gene therapy mediated by scAAV2/8 for dominant retinal disease based on GCAP1 mutation. Further, nonallele-specific RNAi knockdown of GCAP1 may prove generally applicable toward the rescue of any human GCAP1-based dominant cone-rod dystrophy.

Keywords: photoreceptor guanylate cyclase, guanylate cyclase-activating protein 1, short-hairpin RNA, RNA interference, self-complementary adeno-associated virus, cone-rod dystrophy, retinitis pigmentosa

\section{INTRODUCTION}

Cone-rod dystrophies constitute a rare (1/40,000 prevalence) and heterogeneous class of hereditary retinal disease (Hamel, 2007). Symptoms of cone-rod dystrophy (CORD) may include photoaversion, attenuation of central visual acuity, achromatopsia, and eventually, extinction of peripheral vision. To date, ten genes are associated with dominant CORD: PROM1 (Prominin-1), PRPH2 (Peripherin/rds), GUCA1A (GCAP1), RIMS1 (Regulating Synaptic Membrane Exocytosis), GUCY2D (Guanylate Cyclase 1), AIPL1 (Arylhydrocarbon-Interacting receptor ProteinLike 1), PITPNM3 (Phosphatidyl Inositol Transfer Membraneassociated family member 3), UNC119 (Uncoordinated 119 or HRG4), CRX (Cone-Rod otX-like photoreceptor homeobox transcription factor), and SEMA4A (Semaphorin 4A; RETNET at https://sph.uth.edu/retnet/disease.htm). GUCA1A, encoding guanylate cyclase-activating protein 1 (GCAP1), is one of the most fully-characterized dominant CORD genes (Baehr and $\mathrm{Pal}-$ czewski, 2009) and involves about one dozen families with $>100$ affected members harboring various GUCA1A mutations (Jiang et al., 2011).

Guanylate cyclase-activating protein 1 plays a key role in inhibiting photoreceptor guanylate cyclase activity at high free $\mathrm{Ca}^{2+}$, and accelerating guanylate cyclase activity in low free $\mathrm{Ca}^{2+}$. Hydrolysis of cyclic guanosine monophosphate (cGMP) by the phototransduction cascade closes cGMP-gated channels, reducing influx of $\mathrm{Ca}^{2+}$ ions (Figure 1). Continuous extrusion of $\mathrm{Ca}^{2+}$ by the light-insensitive NCKX exchanger lowers cytoplasmic $\mathrm{Ca}^{2+}$, thereby activating GCAP1, and GC and re-establishing dark cGMP levels. In rods, cGMP levels are regulated by two guanylate cyclases (GC1 and GC2; Goraczniak et al., 1994, 1997; Lowe et al., 1995; Duda et al., 1996) and two GCAPs (GCAP1 and GCAP2; Dizhoor et al., 1994, 1995; Palczewski et al., 1994; Gorczyca et al., 1995), while cone phototransduction relies on GC1 and GCAP1 exclusively. The two GCAPs overlap partially in regulating the GCs of rods (Makino et al., 2008; Dizhoor et al., 2010; Peshenko et al., 2011), but with differential signaling modes (Duda et al., 2005, 2012). Both GCAPs contribute to rod recovery after photolysis (Mendez et al., 2001; Howes et al., 2002; Makino et al., 2008, 2012). Germline deletion of both GCAPs renders GC activity in rods and cones $\mathrm{Ca}^{2+}$-insensitive (Mendez et al., 2001). Transgenic GCAP1 could restore normal rod and cone response recovery (Howes et al., 2002; Pennesi et al., 2003).

GCAPs feature four EF hand motifs, of which three (EF2-4) are high-affinity $\mathrm{Ca}^{2+}$ binding sites and one (EF1) is inactive (Palczewski etal., 2004; Baehr and Palczewski, 2009). Six missense mutations in GCAP1 associated with autosomal dominant CORD3 are found in EF3 (E89K, Y99C, D100E, D100G, N104K, I107T; Dizhoor et al., 1998; Payne et al., 1998; Sokal et al., 1998; Jiang et al., 2008; Kitiratschky et al., 2009; Kamenarova et al., 2013; Nong et al., 2014). In EF4, only four missense 


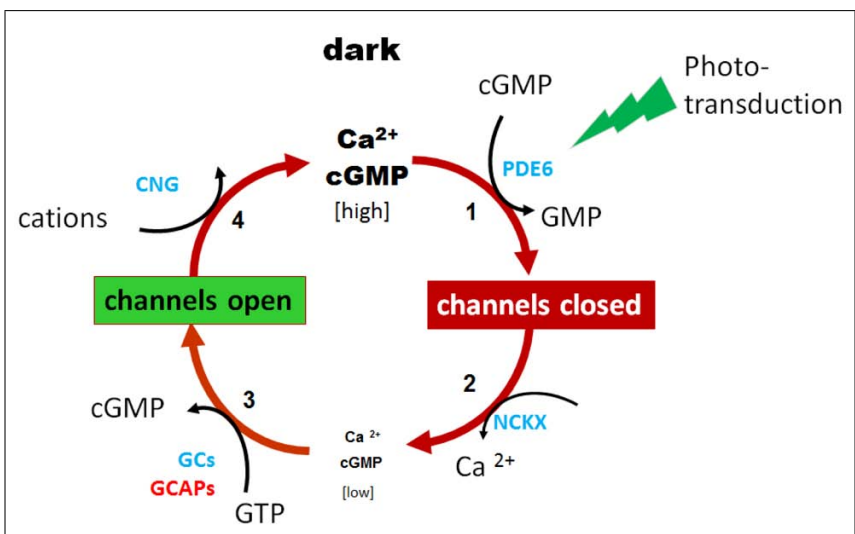

light

FIGURE 1 | Phototransduction feedback loop regulates levels of cGMP and $\mathrm{Ca}^{2+}$. Dark levels of cGMP and $\mathrm{Ca}^{2+}$ are high in rod and cone photoreceptors. Light activation of rhodopsin initiates rod phototransduction, activated PDE6 rapidly hydrolyzes cytoplasmic cGMP, and cGMP-gated cation channels close. Continued extrusion of $\mathrm{Ca}^{2+}$ by the light-insensitive $\mathrm{Na}^{+}-\mathrm{K}^{+} / \mathrm{Ca}^{2+}$ exchanger (NCKX) lowers free $\mathrm{Ca}^{2+}$ which activates guanylate cyclase-activating proteins (GCAPs) and guanylate cyclases (GCs). Restoration of cGMP dark levels re-opens cation channels and $\mathrm{Ca}^{2+}$ levels equilibrate to dark levels.

mutations have been identified (I143NT, L151F, E155G, E155A, G159V; Wilkie etal., 2001; Nishiguchi etal., 2004; Jiang etal., 2005; Sokal etal., 2005; Huang etal., 2013; Figure 2). These dominant GCAP1 mutations alter $\mathrm{Ca}^{2+}$-association, decrease $\mathrm{Ca}^{2+}$ sensitivity, and produce constitutive activity of photoreceptor $\mathrm{GC} 1$ at normal "dark" $\mathrm{Ca}^{2+}$ levels. Persistent stimulation of GC1 in the dark increases cGMP to toxic levels. (Dizhoor etal., 1998; Sokal etal., 1998; Woodruff etal., 2007). Elevated

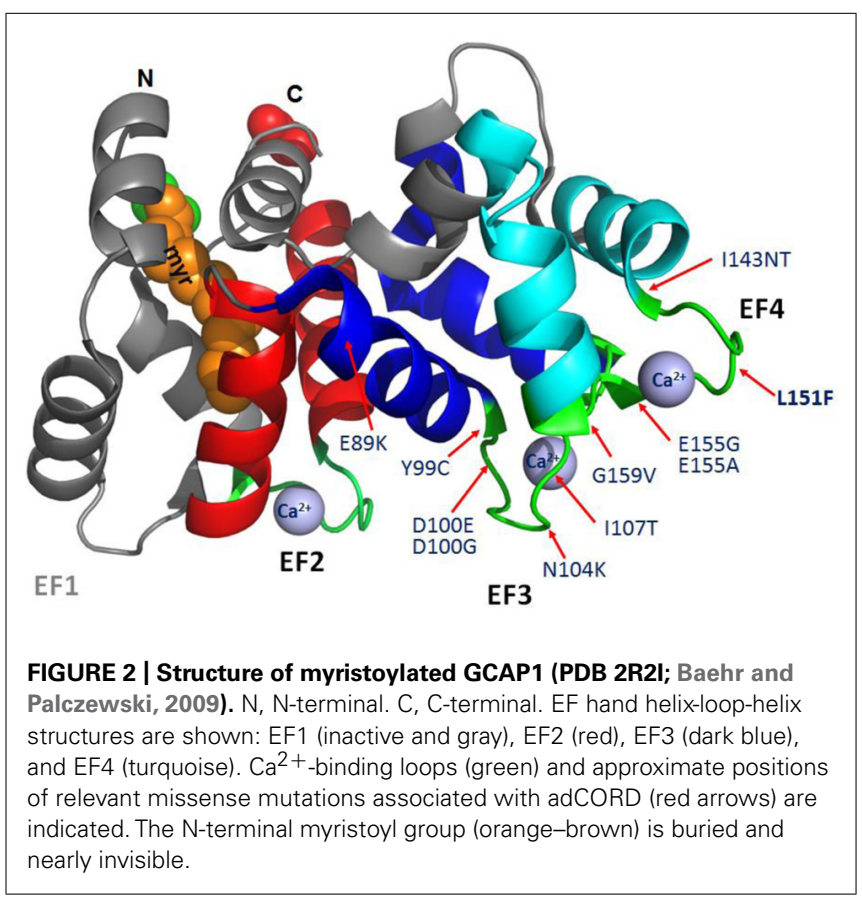

levels of cGMP open more CNG channels, elevate free $\mathrm{Ca}^{2+}$ in outer segments, and trigger cell death by unknown mechanisms. Elevated $\mathrm{Ca}^{2+}$ levels have been suspected to trigger cell death, however a massive accumulation of cGMP in $\mathrm{Cnga3}^{-/-} ; \mathrm{Nrl}^{-/-}$ mice lacking cation channels and elevated $\mathrm{Ca}^{2+}$ also correlated with photoreceptor apoptotic death. This result excludes $\mathrm{Ca}^{2+}$ as a death trigger and supports a role of cGMP accumulation as the major contributor to cone death and a role cGMPdependent protein kinase $\mathrm{G}(\mathrm{PKG})$ regulation in cell death (Xu et al., 2013).

In a four-generation British family, CORD was mapped to chromosome 6p21.1 (Payne et al., 1998). The disease-causing mutation was identified as Y99C, a change that was absent in over 200 unrelated controls. The same mutation was later identified independently in two ancestrally related families (Downes et al., 2001). The Y99C mutation in GCAP1 has also been reported to cause isolated macular dysfunction (Michaelides et al., 2005). Thus far, families presenting with cone and CORD have been independently linked to a GCAP1(L151F) mutation. In the first family (Sokal et al., 2005), hemeralopia, dyschromatopsia and reduced visual acuity became evident by the second-to-third decades of life with non-recordable photopic ERG responses. In the second pedigree spanning five-generations (Jiang et al., 2005), 11 of 24 individuals displayed photoaversion, color vision defects, and central acuity loss with onset of legal blindness during the second-to-third decades of life. The GCAP1(L151F) gene product, known to disrupt $\mathrm{Ca}^{2+}$ coordination at EF-hand four, and alter $\mathrm{Ca}^{2+}$ sensitivity (Sokal et al., 2005), represents a conservative substitution.

\section{GCAP1 MUTANT MOUSE MODELS}

To study GCAP1-associated retinal degeneration disease, several GCAP1 mouse models have been generated, expressing different GCAP1 mutants. The first generated GCAP1 transgenic mice expressing bovine GCAP1(Y99C) mutant gene displayed severe retinitis pigmentosa-like phenotypes as the mutant transgene was specifically expressed in rod photoreceptors under control of a rhodopsin promoter (Olshevskaya et al., 2004). A GCAP1(E155G) knock-in mouse could mimick human patients and presented lateonset and slowly progressive cone-rod photoreceptor degeneration (Buch etal., 2011). Expressing GCAP1(E155G) in mouse rods also caused severe early-onset rod-cone degeneration (Woodruff et al., 2007). Our laboratory generated three transgenic mouse lines expressing wild-type GCAP1-EGFP, as well as mutant GCAP1(L151F), and GCAP1(L151F)-EGFP (Jiang et al., 2013; Figure 3). The three transgenes were modified from mouse genomic Gucala gene fragment, containing its native regulatory elements. They are expressed at a level comparable with endogenous GCAP1 in the transgenic mice, and the mutant transgenic mice develop retina pathology slowly and recapitulate features of human CORD (Jiang et al., 2013).

Our aim was to develop first a self-complementary AAV2/8 (scAAV2/8) knockdown virus that expresses an allele-specific short hairpin RNA (shRNA) targeting the mutant Gucala gene in the GCAP1(Y99C) transgenic mice. Further, we intended to target both mutant and native Gucala in GCAP1(L151F)EGFP transgenic mice (nonallele-specific shRNA targeting). In 


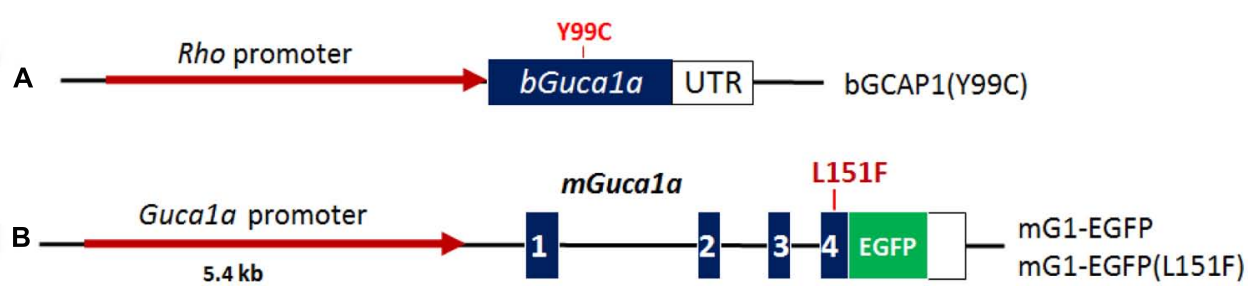

FIGURE 3 | Schematic representation of GCAP1 transgenes used to generate RP and CORD mouse models. (A), bovine GCAP1(Y99C) transgene. Bovine Guca1a cDNA (blue) is expressed under the control of the Rho promoter (red). Approximate position of the Y99C mutation in EF3 is indicated (Jiang et al., 2011). (B), mouse GCAP1 transgene under the control of its native promoter (red). Exons (blue) are numbered; the L151F mutation is located in exon 4 and EGFP is fused in-frame to the GCAP1 C-terminus [see Figure 2 (Jiang et al., 2013)]. the first set, we used two transgenic mouse lines expressing bGCAP1(Y99C) cDNA under the control of the rhodopsin promoter (Figure 3). One line expressed GCAP1 at near normal levels and the second line overexpressed GCAP1 (3-4X; Olshevskaya et al., 2004). These lines are models for dominant retinitis pigmentosa representing moderate (line L52H) or severe (line L53) retinal degeneration phenotypes (Olshevskaya et al., 2004). An allele-specific knockdown bGCAP1(Y99C) is expected to significantly slow down the dystrophy and even delay onset of degeneration.
The second set of transgenic mice contained the L151F mutation introduced in exon 4 of the Gucala gene. We employed the entire Gucala gene including promoter, all exons and introns, and the 3 '-UTR containing the polyadenylation signals. We established two mutant GCAP1 mouse lines, GCAP1(L151F), and GCAP1(L151F)-GFP (Figure 3). A third line expressing a GCAP1EGFP fusion protein (no L151 mutation) served as a WT control (Figure 3). The EGFP tag allowed detection of transgene expression by live fluorescence microscopy, and distinction of transgenic GCAP1-EGFP (50 kDa) from native GCAP1 (23 kDa). We

\begin{tabular}{|c|c|c|c|}
\hline &
\end{tabular}




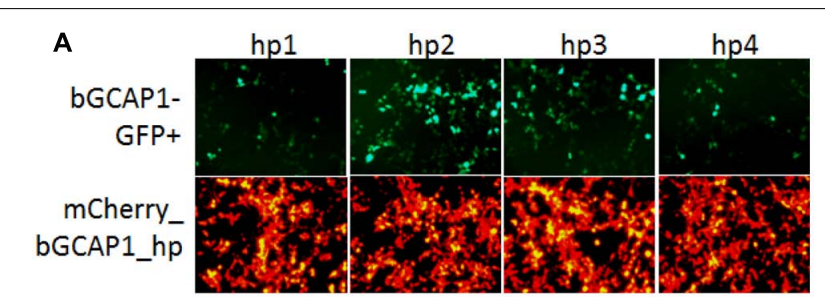

B
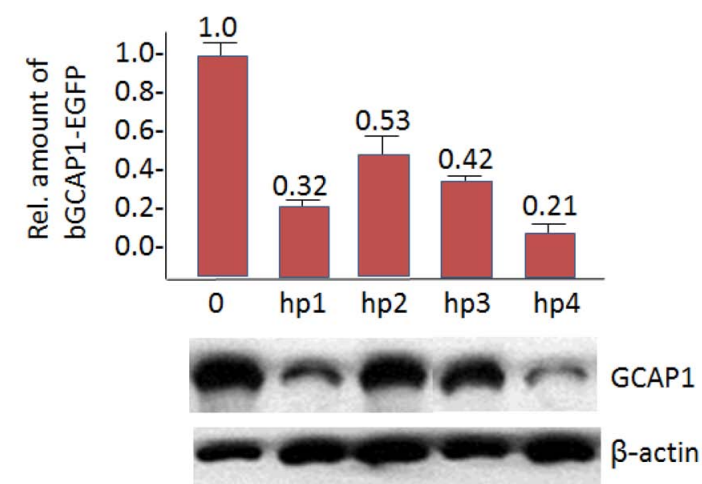

FIGURE 5 | In vitro knockdown of bGCAP1-EGFP. (A), In vitro transfection of HEK cells expressing bGCAP1-GFP (top) with bG1 hp1-4 (bottom). Top panels show that hp1 and hp4 are highly effective. Bottom panels

demonstrate fairly uniform transfection of four shRNA expression plasmids. (B), Representative semi-quantitative immunoblot of GCAP1 knockdown. Bar graph (top, $n=3$ ) identifies hp4 as the most potent shRNA. See also Figure 2 (Jiang et al., 2011), and Figure 7 (Jiang et al., 2013).

intended to knockdown both WT and mutant GCAP1s as removal of photoreceptor GCAP1 does not affect retina development or morphology, and GCAP1/2 double knockout mice do not exhibit retina degeneration.

\section{IN VITRO SCREENING FOR POTENT shRNA SEOUENCES}

For successful knockdown via shRNA, it is essential to screen candidate knockdown sequences for specificity and efficacy. We developed an in vitro screening strategy consisting of a shRNA expression vector with reporter gene, to be co-expressed with a second vector expressing GCAP1-GFP as a prey (Figures 4A,B). This in vitro screening system permits the testing of a number of candidates and eventual identification of the most powerful shRNA. The shRNA vector, hH1pro, contained an shRNA expression cassette driven by a human $\mathrm{H} 1$ pol-III promoter, and a CMV-driven mCherry reporter gene arranged in a tail-to-tail array (Figure 4B). For allelespecific targeting we generated four shRNA constructs, bovine (b) G1hp1-4, expressing four candidate anti-bovine GCAP1 shRNAs specifically targeting bGucala mRNA (Figure 4C). Similarly, we generated four anti-mouse GCAP1 shRNA expression constructs, mGlhpl-4, for nonallele-specific targeting of both transgenic and endogenous mouse GCAP1 mRNAs. (Figure 4D).

In HEK293 cell culture, we tested the knockdown efficiencies of four putative bGCAP1 shRNA by cotransfection of each expression construct (bG1hp1-4) with the target bGCAP1-EGFP (Figure 5A). The expression levels of bGCAP1-EGFP in the transfected cells were detected by live cell fluorescence microscopy and immunoblotting assay $48-50 \mathrm{~h}$ after cotransfection. Under a similar expression level indicating by the mCherry reporter (Figure 5A, lower panel), four bGCAP1 shRNA candidates suppressed GCAP1-GFP expression at different efficiencies (Figure 5A, upper panel). Among them, bG1hp4 was shown the highest knockdown efficiency, presented by the lowest GFP fluorescence in the cotransfected cell cultures, and 79\% reduction of GCAP1-GFP compared to 68,47 , and $58 \%$ for other three shRNAs in semi-quantitative immunochemistry assay (Figure 5B). By using the same in vitro
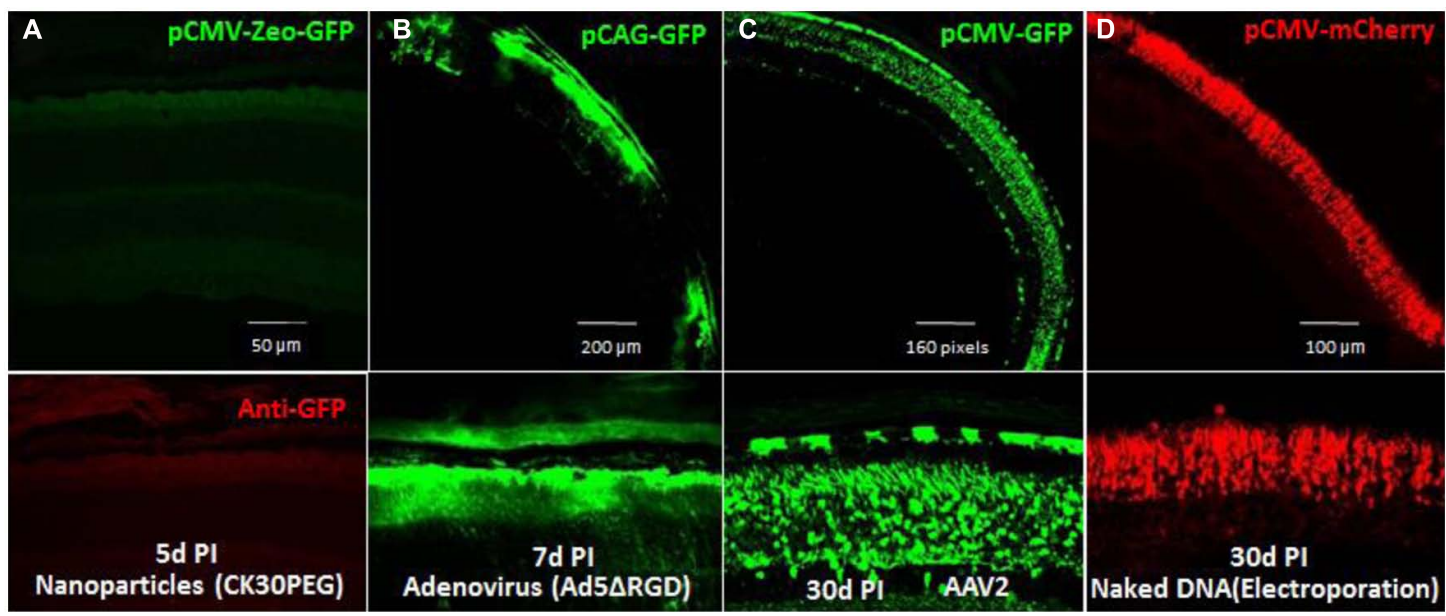

FIGURE 6 | Transduction efficiencies of four gene delivery methods for mouse photoreceptors tested by expression of fluorescent protein reporter. (A), Fluorescence microscopy of retina transversesections of mice injected subretinally with pCMV-Zeo-GFP, peptide/polymer nanoparticles. Below, retina section probed with anti-GFP antibody. (B), Fluorescence microscopy of retina transfected with pCAG-GFP using recombinant adenovirus 5, Ad5 $\triangle \mathrm{RGD}$. Below, section at higher magnification. (C), pCMV-GFP-transfected retina using recombinant $A A V$ vector, $A A V 2$. Underneath, section at higher magnification. (D), pCMV-mCherry-transfected retina using electroporation. Micrographs were recorded at various days post-injection (PI). 


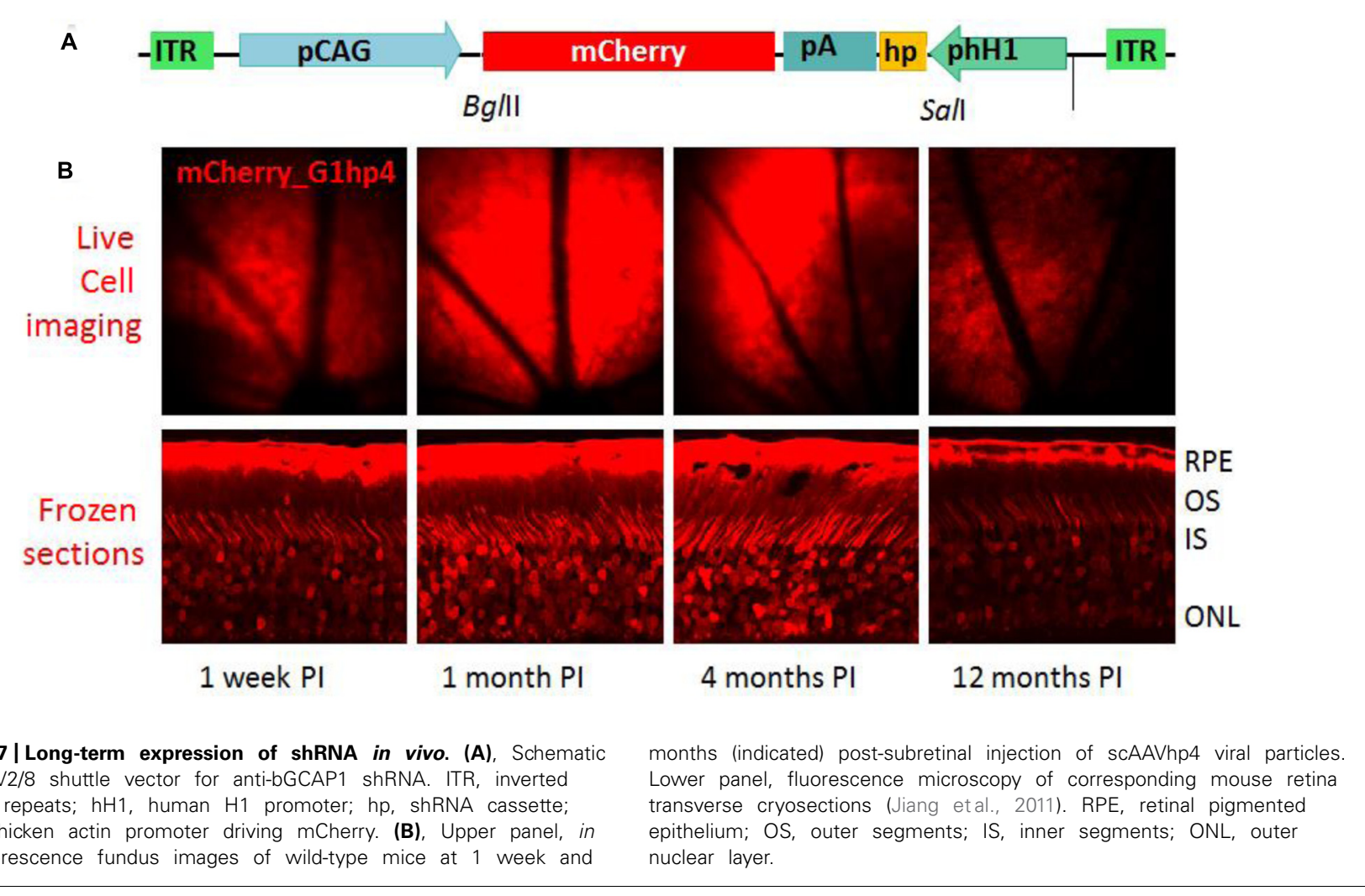

screening experiment, we demonstrated that among four antimouse GCAP1 shRNAs (mGlhp1-4), mG1hp4 is the most efficient, as it deceased mGCAP1-GFP expression by more than 70\% in cell culture. Knockdown specificities of bGlhp4 and mGlhp4 were tested with their 2-nucleotide mismatch shRNA controls, bG1hp4m2 and mG1hp4m2. Compared to bG1hp4 and mGlhp4, the respective mismatch controls were unable to suppress expression of co-transfected bGCAP1-GFP and mGCAP1-GFP (Figure 4).

\section{GENERATION OF SELF-COMPLEMENTARY ADENO-ASSOCIATED VIRUS}

To efficiently express the shRNAs in mouse photoreceptors, we tested four currently used gene delivery vectors: a peptide/polymer nanoparticle (CK30PEG; Farjo et al., 2006), recombinant adenovirus (Ad5 RGD; Cashman et al., 2007; Sweigard et al., 2010), recombinant AAV2 (Wu et al., 2006), and electroporation with naked DNA. No GFP reporter gene expression was detected in the CK30PEG-transduced retina (Figure 6A), and the Ad5 $\triangle$ RGDtransduced retina had much more robust GFP expression in RPE cells than in photoreceptors (Figure 6B). The AAV2-transduced retina showed the most robust GFP expression in photoreceptors (Figure 6C), suggesting AAV vector is a highly efficient gene vector for shRNA delivery to mouse photoreceptors. Although neonatal electroporation of subretinally injected plasmid DNA showed specific and efficient photoreceptor transfection (Figure 6D), this method is only useful for undifferentiated and mitotic photoreceptors and is therefore, not applicable for human gene therapy.
Among currently available AAV vectors, the scAAV vector, scAAV2/8, shows the most effective and stable transgene expression in mouse photoreceptors (Natkunarajah et al., 2008). We packaged the shRNA expression cassette with an mCherry reporter into the recombinant viral vector to generate two scAAV2/8 viral constructs expressing shRNAs targeting bovine and mouse GCAP1s, respectively, scAAVbG1hp4 and scAAVmG1hp4. By examining the mCherry reporter expressed in the scAAVbG1hp4transduced mouse retinas, we demonstrated that ScAAV2/8 vector could generate a long-term transgene expression lasting to 1 year (Figure 7).

\section{THERAPEUTIC EFFECTS OF KNOCKDOWN VIRUS}

To test allele-specific knockdown of GCAP1 in vivo, we injected the scAAVbG1hp4 virus into the mouse subretinal space using two different bGCAP1(Y99C) transgenic mouse lines, L53, and L52H. Expression of the mutant bGCAP1(Y99C), but not that of endogenous mouse GCAP1, was significantly suppressed by scAAV2/8mediated bG1hp4 expression in the retinas of transgenic mice. In the severe and rapid retinal degeneration mouse line L53, bG1hp4 significantly delayed photoreceptor cell death, which was observed at 30 and 45 days post-injection. With the moderate retinal degeneration line $\mathrm{L} 52 \mathrm{H}$, we demonstrated a long-term therapeutic effect of scAAVbG1hp4 virus from 1 month up to 11 months postinjection, assayed by retinal morphology (Figure 8A) and function (Figures 8B,C).

As deletion of GCAPs in mouse apparently has no detrimental morphological nor disease-causing defect, except for increased sensitivity and delayed recovery from the dark state (Mendez 


\section{A}
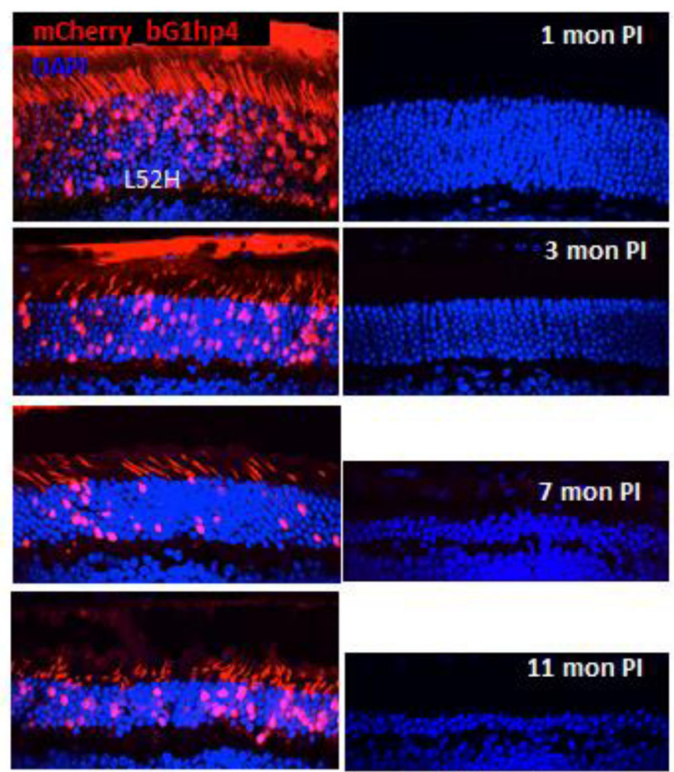

bG1hp4

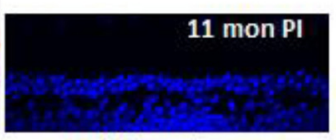

Uninjected

FIGURE 8 | Long-term therapeutic efficiency of allele-specific shRNA vector, scAAV2/8-bG1hp4. (A), Direct fluorescence microscopy of retinal cross-sections examines retinal morphology of the bG1hp4 treated and untreated $\mathrm{L} 52 \mathrm{H}$ transgenic mice. Viral vectors were injected subretinally at mouse ages P21-P30. Both treated and untreated eyes were harvested at four representative times from 1-11 months post-injection. Red, mCherry expression demonstrating scAAV2/8 virus transduction. Blue, DAPI staining

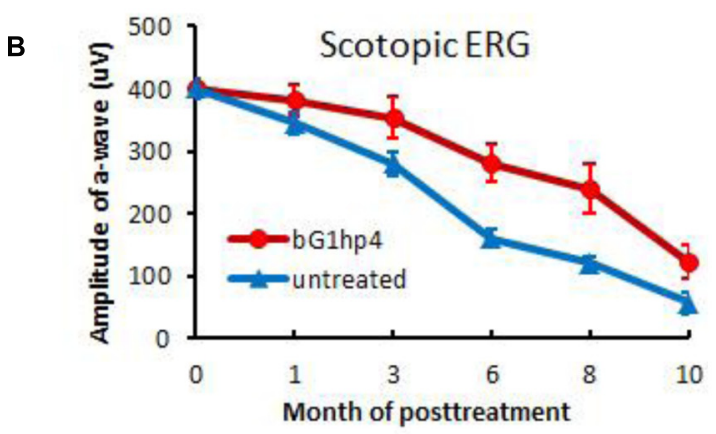

C

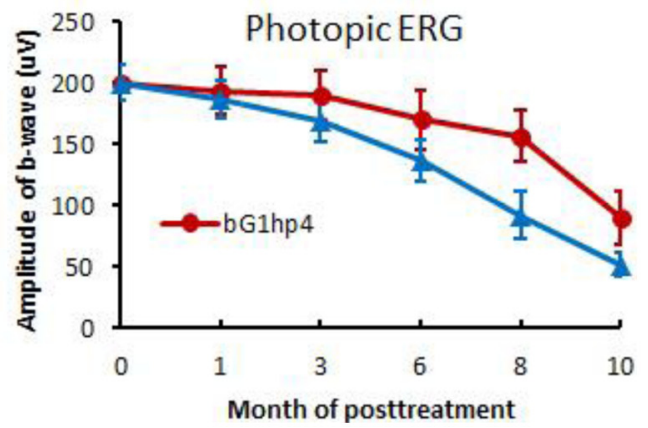

of nuclei. Note significant preservation of ONL thickness at 11 months post-treatment ( 12 months of age) compared to non-treated controls. (B,C). Scotopic and photopic ERG amplitudes recorded from bG1hp4-treated (red) and untreated (blue) L52H transgenic mice (Jiang etal., 2011). Subretinal injection of scAAV2/8-bG1hp4 in the transgenic mouse models delayed progression of both rod and cone dysfunction significantly.

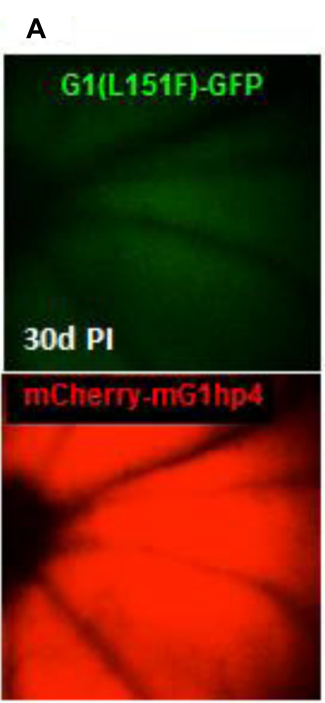

mG1hp4

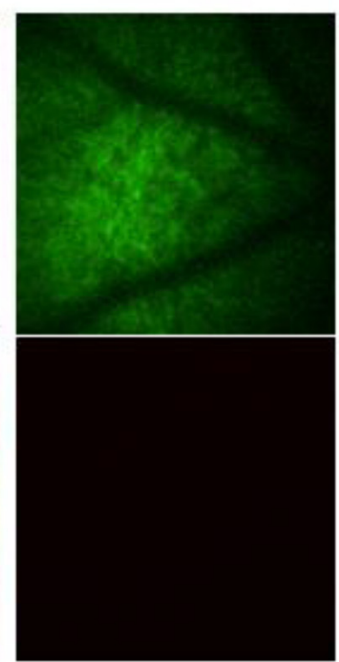

Uninjected
B

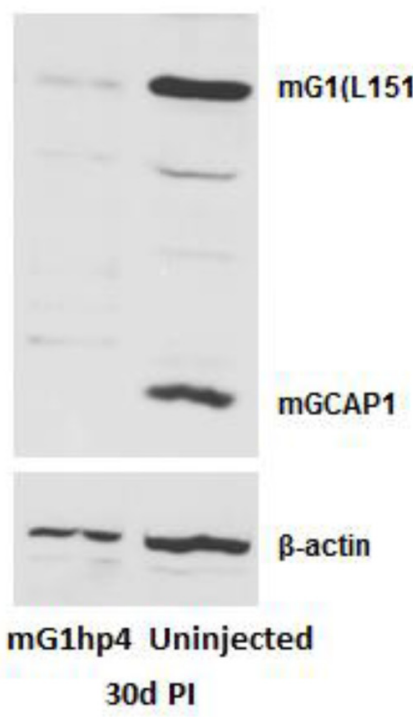

FIGURE 9 | Knockdown of mGCAP1 by nonallele-specific shRNA vector, scAAV2/8-mG1hp4. (A), Fluorescence fundus images of mG1(L151F)-GFP transgenic mice with subretinal injection of the virus vectors at P21-P30 (left) and non-injected control (right). GFP signal (top) represents the $\mathrm{mG1}(\mathrm{L} 151 \mathrm{~F})$-GFP expression level in photoreceptors, and mCherry (bottom) signal indicates scAAV2/8 virus transduction.
(B), Immunoblot of the transgenic mG1(L151F)-GFP and endogenous GCAP1 protein levels in the injected and the non-injected retinas at 30 days PI. Transgenic mG1(L151F)-GFP ( 50 kD) and endogenous GCAP1 ( 25 kD) proteins were detected by UW101 antibody directed against GCAP1. -actin served as endogenous loading control. See also Figure 9 (Jiang etal., 2013). 
etal., 2001), we assumed that nonallele-specific shRNA knockdown of both wild-type and mutant GCAP1s may be a general therapeutic strategy to treat retinal degeneration in patients carrying GCAP1 mutations. We tested in vivo knockdown efficiency of scAAV2/8-mediated mG1hp4 in the mGCAP1(L151F)-GFP transgenic mice. Fluorescence fundus imaging indicated significant suppression of the mGCAP1(L151F)-GFP mutant transgene, but not in uninjected eyes (Figure 9A). Broad and robust mCherry reporter gene expression in scAAVmG1hp4-treated mouse retinas signaled even expression levels of the virus. Semi-quantitative analysis of GCAP1 levels in treated retinas revealed that mG1hp4 simultaneously knocked down in vivo expression of the mutant mGCAP1(L151F)-GFP transgene by 70\% and endogenous wildtype GCAP1 by 90\% (Figure 9B). Thus our experiment provides "proof of principle" that nonallele-specific RNA interference (RNAi) knockdown may be a strategy applicable to all GCAP1 mutations.

\section{CONCLUSION}

The experimental goal was to test whether allele-specific or nonallele-specific knockdown of a dominant GCAP1 mutant is able to ameliorate photoreceptor dystrophy. We demonstrated the feasibility of shRNA knockdown first with an allele-specific approach in a retinitis pigmentosa mouse model expressing GCAP1(Y99C). A scAAV robustly expressed shRNAs in photoreceptors at 1 week post-injection and gene silencing activity persisted as long as 1 year without any apparent off-target interference. Delayed disease onset, significantly improved rod photoreceptor survival and increased visual function support that the methodology can be useful for human gene therapy.

For nonallele-specific knockdown, we generated a sophisticated set of transgenic mouse models expressing GCAP1-EGFP fusion proteins with and without L151F mutation. The L151F mutation, discovered in our lab, was shown to cause dystrophy in two unrelated families. Nonallele-specific shRNA knockdown of both wild-type and mutant GCAP1s may serve as a therapeutic strategy to rescue the dominant degeneration caused by any of the eleven known EF-hand GCAP1 mutations. An advantage of dominant GCAP1 mutations is that a nonalle-specific approach promises to be successful, while mutations in other CORD genes require gene replacement to rescue the disease. Successful knockdown by RNAi suppression of both wild-type and mutant GCAP1s may be a potent therapeutic strategy, applicable to all affected family members with CORD based on GCAP1 mutations in EF3 and $\mathrm{EF} 4$, as long as the shRNA guide strand is located external to the disease-causing mutations.

\section{REFERENCES}

Baehr, W., and Palczewski, K. (2009). Focus on molecules: guanylate cyclase-activating proteins (GCAPs). Exp. Eye Res. 89, 2-3. doi: 10.1016/j.exer.2008.12.016

Buch, P. K., Mihelec, M., Cottrill, P., Wilkie, S. E., Pearson, R. A., Duran, Y., et al. (2011). Dominant cone-rod dystrophy: a mouse model generated by gene targeting of the GCAP1/Gucala gene. PLoS ONE 6:e18089. doi: 10.1371/journal.pone.0018089

Cashman, S. M., McCullough, L., and Kumar-Singh, R. (2007). Improved retinal transduction in vivo and photoreceptor-specific transgene expression using adenovirus vectors with modified penton base. Mol. Ther. 15, 1640-1646. doi: 10.1038/sj.mt.6300203
Dizhoor, A. M., Boikov, S. G., and Olshevskaya, E. V. (1998). Constitutive activation of photoreceptor guanylate cyclase by Y99C mutant of GCAP-1. Possible role in causing human autosomal dominant cone degeneration. J. Biol. Chem. 273, 17311-17314. doi: 10.1074/jbc.273.28.17311

Dizhoor, A. M., Lowe, D. G., Olshevskaya, E. V., Laura, R. P., and Hurley, J. B. (1994). The human photoreceptor membrane guanylyl cyclase, RetGC, is present in outer segments and is regulated by Calcium and a soluble activator. Neuron 12, 1345-1352. doi: 10.1016/0896-6273(94)90449-9

Dizhoor, A. M., Olshevskaya, E. V., Henzel, W. J., Wong, S. C., Stults, J. T., Ankoudinova, I., et al. (1995). Cloning, sequencing, and expression of a $24-\mathrm{kDa}$ Ca2+-binding protein activating photoreceptor guanylyl cyclase. J. Biol. Chem. 270, 25200-25206. doi: 10.1074/jbc.270.42.25200

Dizhoor, A. M., Olshevskaya, E. V., and Peshenko, I. V. (2010). Mg2+/Ca2+ cation binding cycle of guanylyl cyclase activating proteins (GCAPs): role in regulation of photoreceptor guanylyl cyclase. Mol. Cell. Biochem. 334, 117-124. doi: 10.1007/s11010-009-0328-6

Downes, S. M., Holder, G. E., Fitzke, F. W., Payne, A. M., Warren, M. J., Bhattacharya, S. S., etal. (2001). Autosomal dominant cone and cone-rod dystrophy with mutations in the guanylate cyclase activator $1 \mathrm{~A}$ gene-encoding guanylate cyclase activating protein-1. Arch. Ophthalmol. 119, 96-105. doi: 10.1001/archopht.119.11.1667

Duda, T., Fik-Rymarkiewicz, E., Venkataraman, V., Krishnan, R., Koch, K. W., and Sharma, R. K. (2005). The calcium-sensor guanylate cyclase activating protein type 2 specific site in rod outer segment membrane guanylate cyclase type 1 . Biochemistry 44, 7336-7345. doi: 10.1021/bi050068x

Duda, T., Goraczniak, R. M., Surgucheva, I., Rudnicka-Nawrot, M., Gorczyca, W. A., Palczewski, K., etal. (1996). Calcium modulation of bovine photoreceptor guanylate cyclase. Biochemistry 35, 8478-8482. doi: 10.1021/bi96 $0752 \mathrm{z}$

Duda, T., Pertzev, A., and Sharma, R. K. (2012). Differential Ca ${ }^{2+}$ sensor guanylate cyclase activating protein modes of photoreceptor rod outer segment membrane guanylate cyclase signaling. Biochemistry $51,4650-4657$. doi: 10.1021/bi30 $0572 \mathrm{w}$

Farjo, R., Skaggs, J., Quiambao, A. B., Cooper, M. J., and Naash, M. I. (2006). Efficient non-viral ocular gene transfer with compacted DNA nanoparticles. PLoS ONE 1:e38. doi: 10.1371/journal.pone.0000038

Goraczniak, R., Duda, T., and Sharma, R. K. (1997). Structural and functional characterization of a second subfamily member of the calcium-modulated bovine rod outer segment membrane guanylate cyclase, ROS-GC2. Biochem. Biophys. Res. Commun. 234, 666-670. doi: 10.1006/bbrc.1997.6579

Goraczniak, R. M., Duda, T., Sitaramayya, A., and Sharma, R. K. (1994). Structural and functional characterization of the rod outer segment membrane guanylate cyclase. Biochem. J. 302, 455-461.

Gorczyca, W. A., Polans, A. S., Surgucheva, I., Subbaraya, I., Baehr, W., and Palczewski, K. (1995). Guanylyl cyclase activating protein: a calciumsensitive regulator of phototransduction. J. Biol. Chem. 270, 22029-22036. doi: $10.1074 /$ jbc.270.37.22029

Hamel, C. P. (2007). Cone rod dystrophies. Orphanet J. Rare Dis. 2, 7. doi: $10.1186 / 1750-1172-2-7$

Howes, K. A., Pennesi, M. E., Sokal, I., Church-Kopish, J., Schmidt, B., Margolis, D., et al. (2002). GCAP1 rescues rod photoreceptor response in GCAP1/GCAP2 knockout mice. $E M B O$ J. 21, 1545-1554. doi: 10.1093/emboj/21.7.1545

Huang, L., Li, S., Xiao, X., Jia, X., Sun, W., Gao, Y., et al. (2013). Novel GUCA1A mutation identified in a Chinese family with cone-rod dystrophy. Neurosci. Lett. 541, 179-183. doi: 10.1016/j.neulet.2013.02.013

Jiang, L., Katz, B. J., Yang, Z., Zhao, Y., Faulkner, N., Hu, J., et al. (2005). Autosomal dominant cone dystrophy caused by a novel mutation in the GCAP1 gene (GUCA1A). Mol. Vis. 11, 143-151.

Jiang, L., Li, T. Z., Boye, S. E., Hauswirth, W. W., Frederick, J. M., and Baehr, W. (2013). RNAi-mediated gene suppression in a GCAP1(L151F) cone-rod dystrophy mouse model. PLoS ONE 8:e57676. doi: 10.1371/journal.pone.00 57676

Jiang, L., Wheaton, D., Bereta, G., Zhang, K., Palczewski, K., Birch, D. G., et al. (2008). A novel GCAP1(N104K) mutation in EF-hand 3 (EF3) linked to autosomal dominant cone dystrophy. Vision Res. 48, 2425-2432. doi: 10.1016/j.visres.2008.07.016

Jiang, L., Zhang, H., Dizhoor, A. M., Boye, S. E., Hauswirth, W. W., Frederick, J. M., et al. (2011). Long-term RNA interference gene therapy in a dominant retinitis 
pigmentosa mouse model. Proc. Natl. Acad. Sci. U.S.A. 108, 18476-18481. doi: 10.1073/pnas.1112758108

Kamenarova, K., Corton, M., Garcia-Sandoval, B., Fernandez-San, J. P., Panchev, V., Avila-Fernandez, A., et al. (2013). Novel GUCA1A mutations suggesting possible mechanisms of pathogenesis in cone, cone-rod, and macular dystrophy patients. Biomed. Res. Int. 2013, 517570. doi: 10.1155/2013/517570

Kitiratschky, V. B., Behnen, P., Kellner, U., Heckenlively, J. R., Zrenner, E., Jagle, H., et al. (2009). Mutations in the GUCA1A gene involved in hereditary cone dystrophies impair calcium-mediated regulation of guanylate cyclase. Hum. Mutat. 30, E782-E796. doi: 10.1002/humu.21055

Lowe, D. G., Dizhoor, A. M., Liu, K., Gu, Q., Spencer, M., Laura, R., et al. (1995). Cloning and expression of a second photoreceptor-specific membrane retina guanylyl cyclase (RetGC), RetGC-2. Proc. Natl. Acad. Sci. U.S.A. 92, 5535-5539. doi: 10.1073/pnas.92.12.5535

Makino, C. L., Peshenko, I. V., Wen, X. H., Olshevskaya, E. V., Barrett, R., and Dizhoor, A. M. (2008). A role for GCAP2 in regulating the photoresponse. Guanylyl cyclase activation and rod electrophysiology in GUCA1B knock-out mice. J. Biol. Chem. 283, 29135-29143. doi: 10.1074/jbc.M804445200

Makino, C. L., Wen, X. H., Olshevskaya, E. V., Peshenko, I. V., Savchenko, A. B., and Dizhoor, A. M. (2012). Enzymatic relay mechanism stimulates cyclic GMP synthesis in rod photoresponse: biochemical and physiological study in guanylyl cyclase activating protein 1 knockout mice. PLOS ONE 7:e47637. doi: 10.1371/journal.pone.0047637

Mendez, A., Burns, M. E., Sokal, I., Dizhoor, A. M., Baehr, W., Palczewski, K., et al. (2001). Role of guanylate cyclase-activating proteins (GCAPs) in setting the flash sensitivity of rod photoreceptors. Proc. Natl. Acad. Sci. U.S.A. 98, 9948-9953. doi: 10.1073/pnas.171308998

Michaelides, M., Wilkie, S. E., Jenkins, S., Holder, G. E., Hunt, D. M., Moore, A. T., etal. (2005). Mutation in the gene GUCA1A, encoding guanylate cyclase-activating protein 1, causes cone, cone-rod, and macular dystrophy. Ophthalmology 112, 1442-1447. doi: 10.1016/j.ophtha.2005.02.024

Natkunarajah, M., Trittibach, P., McIntosh, J., Duran, Y., Barker, S. E., Smith, A. J., et al. (2008). Assessment of ocular transduction using single-stranded and selfcomplementary recombinant adeno-associated virus serotype 2/8. Gene Ther. 15 463-467. doi: 10.1038/sj.gt.3303074

Nishiguchi, K. M., Sokal, I., Yang, L., Roychowdhury, N., Palczewski, K., Berson, E. L., et al. (2004). A novel mutation (I143NT) in guanylate cyclaseactivating protein 1 (GCAP1) associated with autosomal dominant cone degeneration. Invest. Ophthalmol. Vis. Sci. 45, 3863-3870. doi: 10.1167/iovs. 04-0590

Nong, E., Lee, W., Merriam, J. E., Allikmets, R., and Tsang, S. H. (2014). Disease progression in autosomal dominant cone-rod dystrophy caused by a novel mutation (D100G) in the GUCA1A gene. Doc. Ophthalmol. 128, 59-67. doi: 10.1007/s10633-013-9420-z

Olshevskaya, E. V., Calvert, P. D., Woodruff, M. L., Peshenko, I. V., Savchenko, A. B., Makino, C. L., et al. (2004). The Y99C mutation in guanylyl cyclase-activating protein 1 increases intracellular $\mathrm{Ca}^{2+}$ and causes photoreceptor degeneration in transgenic mice. J. Neurosci. 24, 6078-6085. doi: 10.1523/JNEUROSCI.096304.2004

Palczewski, K., Sokal, I., and Baehr, W. (2004). Guanylate cyclase-activating proteins: structure, function, and diversity. Biochem. Biophys. Res. Commun. 322, 1123 1130. doi: 10.1016/j.bbrc.2004.07.122

Palczewski, K., Subbaraya, I., Gorczyca, W. A., Helekar, B. S., Ruiz, C. C., Ohguro, H., et al. (1994). Molecular cloning and characterization of retinal photoreceptor guanylyl cyclase activating protein (GCAP). Neuron 13, 395-404. doi: 10.1016/0896-6273(94)90355-7
Payne, A. M., Downes, S. M., Bessant, D. A., Taylor, R., Holder, G. E., Warren, M. J., et al. (1998). A mutation in guanylate cyclase activator 1A (GUCA1A) in an autosomal dominant cone dystrophy pedigree mapping to a new locus on chromosome 6p21.1. Hum. Mol. Genet. 7, 273-277. doi: 10.1093/hmg/7. 2.273

Pennesi, M. E., Howes, K. A., Baehr, W., and Wu, S. M. (2003). Guanylate cyclase-activating protein (GCAP) 1 rescues cone recovery kinetics in GCAP1/GCAP2 knockout mice. Proc. Natl. Acad. Sci U.S.A. 100, 6783-6788. doi: 10.1073/pnas.1130102100

Peshenko, I. V., Olshevskaya, E. V., Savchenko, A. B., Karan, S., Palczewski, K., Baehr, W., et al. (2011). Enzymatic properties and regulation of the native isozymes of retinal membrane guanylyl cyclase (RetGC) from mouse photoreceptors. Biochemistry 50, 5590-5600. doi: 10.1021/bi200491b

Sokal, I., Dupps, W. J., Grassi, M. A., Brown, J. Jr., Affatigato, L. M., Roychowdhury, N., et al. (2005). A GCAP1 missense mutation (L151F) in a large family with autosomal dominant cone-rod dystrophy (adCORD). Invest. Ophthalmol. Vis. Sci. 46, 1124-1132. doi: 10.1167/iovs.04-1431

Sokal, I., Li, N., Surgucheva, I., Warren, M. J., Payne, A. M., Bhattacharya, S. S., et al. (1998). GCAP1(Y99C) mutant is constitutively active in autosomal dominant cone dystrophy. Mol. Cell 2, 129-133. doi: 10.1016/S1097-2765(00) 80121-5

Sweigard, J. H., Cashman, S. M., and Kumar-Singh, R. (2010). Adenovirus vectors targeting distinct cell types in the retina. Invest. Ophthalmol. Vis. Sci. 51, 22192228. doi: 10.1167/iovs.09-4367

Wilkie, S. E., Li, Y., Deery, E. C., Newbold, R. J., Garibaldi, D., Bateman, J. B., et al. (2001). Identification and functional consequences of a new mutation (E155G) in the gene for GCAP1 that causes autosomal dominant cone dystrophy. Am. J. Hum. Genet. 69, 471-480. doi: 10.1086/323265

Woodruff, M. L., Olshevskaya, E. V., Savchenko, A. B., Peshenko, I. V., Barrett, R., Bush, R. A., et al. (2007). Constitutive excitation by Gly90Asp rhodopsin rescues rods from degeneration caused by elevated production of cGMP in the dark. J. Neurosci. 27, 8805-8815. doi: 10.1523/JNEUROSCI.2751-07.2007

Wu, Z., Asokan, A., and Samulski, R. J. (2006). Adeno-associated virus serotypes: vector toolkit for human gene therapy. Mol. Ther. 14, 316-327. doi: 10.1016/j.ymthe.2006.05.009

Xu, J., Morris, L., Thapa, A., Ma, H., Michalakis, S., Biel, M., et al. (2013). cGMP accumulation causes photoreceptor degeneration in CNG channel deficiency: evidence of cGMP cytotoxicity independently of enhanced CNG channel function. J. Neurosci. 33, 14939-14948. doi: 10.1523/JNEUROSCI.090913.2013

Conflict of Interest Statement: The authors declare that the research was conducted in the absence of any commercial or financial relationships that could be construed as a potential conflict of interest.

Received: 21 January 2014; accepted: 19 March 2014; published online: 07 April 2014. Citation: Jiang L, Frederick JM and Baehr W (2014) RNA interference gene therapy in dominant retinitis pigmentosa and cone-rod dystrophy mouse models caused by GCAP1 mutations. Front. Mol. Neurosci. 7:25. doi: 10.3389/fnmol.2014.00025 This article was submitted to the journal Frontiers in Molecular Neuroscience. Copyright (@) 2014 Jiang, Frederick and Baehr. This is an open-access article distributed under the terms of the Creative Commons Attribution License (CC BY). The use, distribution or reproduction in other forums is permitted, provided the original author(s) or licensor are credited and that the original publication in this journal is cited, in accordance with accepted academic practice. No use, distribution or reproduction is permitted which does not comply with these terms. 